\title{
A TURBULENCE-DRIVEN MODEL FOR HEATING AND ACCELERATION OF THE FAST WIND IN CORONAL HOLES
}

\author{
A. Verdini ${ }^{1}$, M. Velli ${ }^{2,3}$, W. H. Matthaeus ${ }^{4}$, S. Oughton $^{5}$, and P. Dmitruk ${ }^{6}$ \\ ${ }^{1}$ Observatoire Royale de Belgique, 3 Avenue Circulaire, 1180, Bruxelles, Belgium; verdini@ oma.be \\ ${ }^{2}$ Dipart. di Astronomia e Scienza dello Spazio, Univ. di Firenze, Largo E. Fermi 3, 50125, Firenze, Italy \\ 3 Jet Propulsion Laboratory, California Institute of Technology, 4800 Oak Grove Drive, Pasadena, CA 91109, USA \\ ${ }^{4}$ Department of Physics and Astronomy, University of Delaware, DE 19716, USA \\ 5 Department of Mathematics, University of Waikato, Hamilton, New Zealand \\ ${ }^{6}$ Depart. Física, Facultad de Ciencias Exactas y Naturales, Univ. de Buenos Aires-Conicet, Argentina \\ Received 2009 October 15; accepted 2009 November 25; published 2009 December 22
}

\begin{abstract}
A model is presented for generation of fast solar wind in coronal holes, relying on heating that is dominated by turbulent dissipation of MHD fluctuations transported upward in the solar atmosphere. Scale-separated transport equations include large-scale fields, transverse Alfvénic fluctuations, and a small compressive dissipation due to parallel shears near the transition region. The model accounts for proton temperature, density, wind speed, and fluctuation amplitude as observed in remote sensing and in situ satellite data.
\end{abstract}

Key words: MHD - solar wind - turbulence - waves

Online-only material: color figures

\section{INTRODUCTION}

An open question in solar and heliospheric physics is to identify the physical processes responsible for heating the corona and accelerating the fast solar wind streams emanating from coronal holes. This requires that a fraction of the energy available in photospheric motions be transported through the chromospheric transition region, and dissipated in the corona. The measured speeds of fast solar wind streams require spatially extended heating (Withbroe \& Noyes 1977; Holzer \& Leer 1980; Withbroe 1988). The physical mechanisms for this transport and dissipation have remained elusive. Some models have resorted to use of a parametrically defined heat deposition (a "heat function") that decays exponentially with height, or anomalous heat conduction that redistributes energy along field lines (Habbal et al. 1995; McKenzie et al. 1995; Banaszkiewicz et al. 1998). One-dimensional (1D) models of this kind, extending from the chromosphere to $1 \mathrm{AU}$ (Hansteen et al. 1994, 1997), have helped in understanding the regulation of the solar wind mass flux and can reproduce fast solar wind streams originating in cool electron coronal holes.

Here we present a model that demonstrates solar wind acceleration due to heating by a quasi-incompressible turbulent cascade triggered by coronal stratification (Matthaeus et al. 1999), and supplemented by compressive heating near the base. This model accounts for most presently available coronal and interplanetary observations.

The idea that broadband plasma fluctuations might heat the extended corona and accelerate the solar wind has long been discussed (Coleman 1968; Belcher \& Davis 1971; Hollweg 1986; Hollweg \& Johnson 1988; Velli 1993). However, the mechanisms of transfer of fluctuation energy to small scalesand, in particular, the role of Alfvénic turbulence (as observed in the solar wind) and cascade processes (e.g., phase mixing, ponderomotive driving, shocks, etc.) - have not been described self-consistently. Two recent papers shed light on these relationships (Suzuki \& Inutsuka 2005; Cranmer et al. 2007).
Suzuki \& Inutsuka (2005) incorporate 1D compressive nonlinear interactions driven by Alfvén waves and leading to shock heating. This model produces good agreement with solar wind speed profiles. As low-frequency Alfvén waves propagate upward, their wave pressure compresses the plasma. Unable to refract or mode-mode couple into a perpendicular wavenumber cascade, these waves must dissipate in 1D shock fronts. This model provides a valuable demonstration that MHD fluctuations can act as a conduit to transport energy to the requisite altitudes. However, the restriction to 1D cascade is at odds with the well-established propensity for an incompressible MHD cascade to proceed mainly through wavevectors perpendicular to a strong mean magnetic field (Robinson \& Rusbridge 1971; Shebalin et al. 1983; Oughton et al. 1994; Bieber et al. 1996; Cho et al. 2002). Furthermore, the corona exhibits a clear transverse structuring, and the initial fluctuations must have perpendicular correlation lengths not much larger than a supergranulation scale $(15,000 \mathrm{~km})$.

Another recent model (Cranmer \& van Ballegooijen 2005; Cranmer et al. 2007) incorporates a low-frequency cascade model (Verdini et al. 2006); however, the treatment of propagation and dissipation differs significantly from the present approach. Their scheme treats nonlinear effects as a perturbation, and it is unclear if it converges for strong turbulent heating. Here we employ a strong turbulence closure. We also do not rely on electron heat conduction to boost radial energy transport. Instead, we compute an internal energy associated with the protons only. This approach supports comparisons with results employing improved representation of turbulence, such as shell models (Verdini et al. 2009a, 2009b) and (potentially) full MHD simulation.

We find here that reflection of Alfvénic turbulence alone does not lead to a full corona/solar wind stationary statea compressible contribution is required. This is supported by recent observations from Hinode (Langangen et al. 2008; DePontieu et al. 2009). When we include a small component of compressive heating near the coronal base, fast solar wind streams are then accounted for. 


\section{MODEL DESCRIPTION AND EQUATIONS}

We employ a 1D large-scale steady-state MHD model in an expanding flux tube (cf. Leer et al. 1982; Verdini et al. 2006), comprising equations of mass continuity, radial momentum conservation, and pressure (internal energy),

$$
\begin{gathered}
\frac{d}{d r}[\rho U A]=0, \\
\rho U \frac{d U}{d r}=-\nabla P^{\prime}-\rho \frac{G M_{\mathrm{sun}}}{r^{2}}+\mathrm{R}_{r}, \\
U \frac{d p}{d r}=-\gamma p \nabla \cdot \boldsymbol{U}+(\gamma-1) Q(r) .
\end{gathered}
$$

Here $r$ is the radial coordinate, $A(r)$ is the flux tube crosssectional area, $p(r)=2 n k_{\mathrm{B}} T$ is the thermal pressure, $\boldsymbol{U}=$ $U(r) \hat{\boldsymbol{r}}$ is the large-scale radial flow (wind) velocity, $G$ is the gravitational constant, and $M_{\text {sun }}$ is the solar mass. $\mathrm{R}_{r}$ is the radial component of the (vector) divergence of the MHD Reynolds stress $\mathbf{R}=\langle\delta \boldsymbol{b} \cdot \nabla \delta \boldsymbol{b} / 4 \pi-\rho \delta \boldsymbol{u} \cdot \nabla \delta \boldsymbol{u}\rangle$, where $\delta \boldsymbol{u}, \delta \boldsymbol{b}$ are the fluctuations and the full magnetic field is $\boldsymbol{B}=\hat{\boldsymbol{r}} B_{r}(r)+\delta \boldsymbol{b}$, and $\hat{\boldsymbol{r}} \cdot \delta \boldsymbol{b}=0=\hat{\boldsymbol{r}} \cdot \delta \boldsymbol{u}$.

The total (thermal plus magnetic) pressure is

$$
P^{\prime}=2 n k_{\mathrm{B}} T+\frac{\delta \boldsymbol{b}^{2}}{8 \pi}
$$

We specify an area expansion factor $A(r)$. Then $B_{r}(r)$ is determined by magnetic flux conservation, $B_{r}(r) A(r)=$ const, which is constrained by 1 AU observations.

$Q(r)$ is the heating per unit volume, related to the heat function (per unit mass) $H=Q / \rho$. It involves an incompressible part $H_{i}$, associated with turbulence, modeled here in KármánTaylor fashion (e.g., de Kármán \& Howarth 1938; Matthaeus et al. 2004). There is also a small compressive part $H_{c}=Q_{c} / \rho$, so that $H=H_{i}+H_{c}$. Turbulence influences the large-scale flow through $Q_{i}$, wave pressure, and the Reynolds stress $\mathrm{R}_{r}$, and is modeled using only a few free parameters.

The dominant contributor to the turbulent heating, the lowfrequency quasi-incompressible turbulence, is evolved using a transport equation and one-point closure that depends upon the cross helicity. The nonlinear phenomenological model (Dmitruk et al. 2001) involves the Elsässer variables $\mathbf{z}^{ \pm}=\delta \boldsymbol{u} \mp \delta \boldsymbol{b} / \sqrt{4 \pi \rho}$, their associated energies $E^{ \pm}=Z_{ \pm} / 4=\left\langle\left|\mathbf{z}^{ \pm}\right|^{2}\right\rangle / 4,(\langle\cdots\rangle$ indicates an ensemble average), and a common similarity (correlation) scale $\lambda$. The dimensionless cross helicity $\sigma_{c}=$ $\left(Z_{+}^{2}-Z_{-}^{2}\right) /\left(Z_{+}^{2}+Z_{-}^{2}\right)$ measures any excess inward or outward propagating-type fluctuations. The incompressible turbulent heating model (Hossain et al. 1995) is

$$
H_{i}(r)=\frac{Q_{i}(r)}{\rho} \equiv \frac{1}{2} \frac{Z_{-} Z_{+}^{2}+Z_{+} Z_{-}^{2}}{\lambda} .
$$

See also Dobrowolny et al. (1980), Grappin et al. (1983), and Matthaeus et al. (2004).

We include spatial transport (Verdini \& Velli 2007) in a nonuniform wind with speed $U(r) \hat{\boldsymbol{r}}$. The fluctuations are assumed to be Reduced MHD-like (i.e., perpendicular fluctuations, parallel gradients much weaker than transverse ones) and represented by "typical amplitudes" of a given frequency $z_{ \pm}(\omega)$, defined such that $Z_{ \pm}^{2}=\int_{\Omega}\left(z_{ \pm}^{2} / \omega\right) \mathrm{d} \omega$ :

$$
\left[U \pm V_{a}\right] \frac{d z_{ \pm}}{d r}+i \omega z_{ \pm}=R_{1}^{ \pm} z_{ \pm}+R_{2}^{ \pm} z_{\mp}-\frac{\left|Z_{\mp}\right|}{2 \lambda} z_{ \pm} .
$$

The WKB (Equation (7)) and reflection (Equation (8)) terms are related to large-scale gradients by

$$
\begin{gathered}
R_{1}^{ \pm}=-\frac{1}{2}\left[U \mp V_{a}\right]\left(\frac{d \log V_{a}}{d r}+\frac{d \log A}{d r}\right), \\
R_{2}^{ \pm}=\frac{1}{2}\left[U \mp V_{a}\right] \frac{d \log V_{a}}{d r} .
\end{gathered}
$$

Here $V_{a}=V_{a_{r}}=B_{r} / \sqrt{4 \pi \rho}$. For simplicity the lengthscale $\lambda$ is associated with the expansion, using $\lambda(r)=\lambda_{\odot} \sqrt{A(r)}$. The $\odot$ subscript indicates evaluation at the coronal base, here taken to be at the top of the transition region.

The smaller, compressive contribution to the heating $\left(H_{c}\right)$ is assumed to be confined near the coronal base where it rapidly dissipates through shocks. This is motivated by recent observations (Langangen et al. 2008; DePontieu et al. 2009) of fluctuations with parallel (vertical) variance that pervade the entire corona near the transition region. Here we model this effect directly as a heat function, and assume that it contributes $\approx 1 \%$ of the total (height-integrated) heat function.

Having in hand the complete set of Equations (1)-(3), and (6), along with constitutive relations (4), (5), (7), and (8), and the small compressive heat function $H_{c}$, we are in position to solve a relatively complete and self-consistent solar wind model with turbulence. The stationary solutions for the large-scale fields and turbulence parameters are obtained via a numerical iteration procedure in which $T_{\odot}=4 \times 10^{5} \mathrm{~K}$ and $n_{\odot}=5 \times 10^{8} \mathrm{~cm}^{-3}$ are held constant. To begin, we impose trial radial profiles for $T$ and $n$, and then solve Equations (6) with a fixed amplitude of the velocity fluctuation at the base, say $\delta u_{\odot}=30 \mathrm{~km} \mathrm{~s}^{-1}$. From this temporary solution we compute the Reynolds stress, the ponderomotive force, and the heating function that appear in Equations (1)-(3). Next, those equations are solved for $T$ and $U$, which provides updated values for $V_{a}, U$, and their derivatives. These updated profiles are used in re-solving Equations (6), and so on.

After few iterations, there are small relative differences $\left(\lesssim 10^{-5}\right)$ in each of the large-scale and fluctuation fields. We control convergence through the relative variation of the positions of the sonic and Alfvénic critical points, assuming convergence when $\Delta r_{s} / r_{s}, \Delta r_{a} / r_{a}<10^{-4}$. For each frequency $\omega$, we solve subject to imposed conditions on $\delta u_{\odot}, H_{c}(r)$, the maximal (over)expansion $f_{\max }=\max \left[A(r) / r^{2}\right]$, and the correlation length scale $\lambda_{\odot}$. We use, as in Kopp \& Holzer (1976), $f(r)=A(r) / r^{2}$, with

$$
f(r)=\frac{f_{\max } \exp \left[\left(r-r_{f}\right) / \sigma\right]+f_{1}}{\exp \left[\left(r-r_{f}\right) / \sigma\right]+f_{1}},
$$

$f_{1}=1-f_{\max } \exp \left[\left(R_{\odot}-r_{f}\right) / \sigma\right]$, keeping fixed the location and width of the super-radial expansion, $r_{f}=1.31 R_{\odot}$ and $\sigma=0.51 R_{\odot}$, respectively.

\section{RESULTS}

A typical solution is shown in Figure 1 (solid black line), where we plot several quantities $\left(U, T, n, \sigma_{c}, Z^{2}\right)$ as a function of distance, obtained with the chosen parameters: $\delta u_{\odot}=$ $30 \mathrm{~km} \mathrm{~s}^{-1}, \omega=0 \mathrm{~Hz}, \lambda_{\odot}=0.015 R_{\odot}, f_{\max }=12.5$, and $H_{c}=1 \% H$. The speed $U$, temperature $T$, and density $n$ are generally in good agreement with the observations in the heliosphere, although the terminal speed is about $100 \mathrm{~km} \mathrm{~s}^{-1}$ higher and $T$ peaks too far from the coronal base compared 

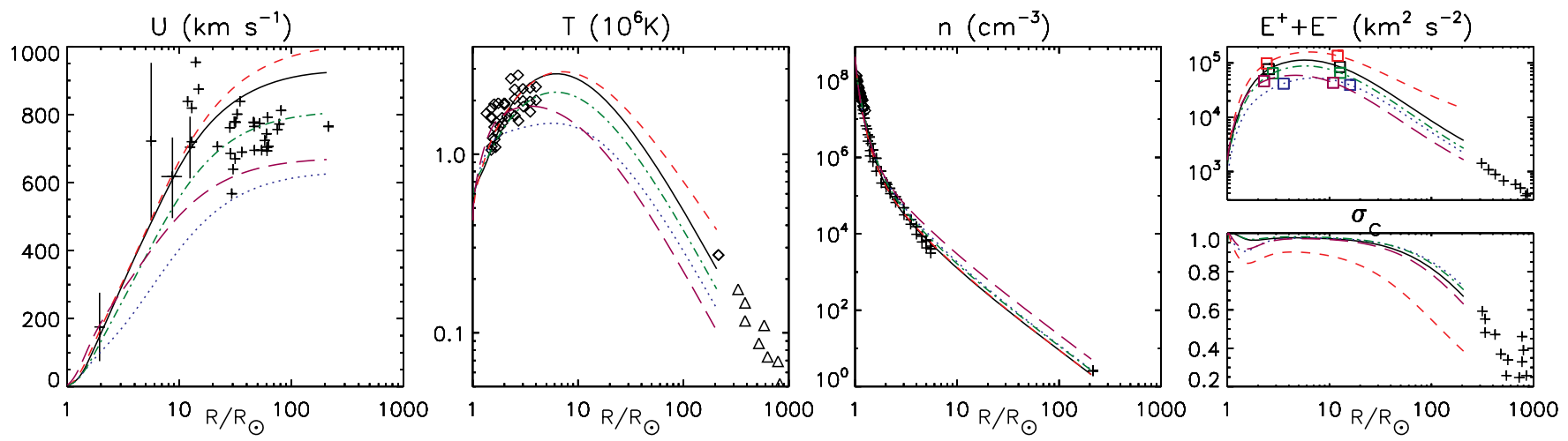

Figure 1. Wind speed, temperature, density, turbulence level, and normalized cross helicity as a function of distance for the representative solution (solid line) with $\delta u_{\odot}=30 \mathrm{~km} \mathrm{~s}^{-1}, \omega=0 \mathrm{~Hz}, \lambda_{\odot}=0.015 R_{\odot}, f_{\max }=12.5$, and $H_{c}=1 \% H$. The other lines denote solutions for which one of the parameters has been varied; specifically, $\delta u_{\odot}=20 \mathrm{~km} \mathrm{~s}^{-1}, \lambda_{\odot}=0.05 R_{\odot}, f_{\max }=10$, and $H_{c} \approx 5 \% H$, in blue dotted, red dashed, green dashed-dotted, violet long-dashed lines, respectively. Empty squares in the top-right panel mark the position of $r_{s}$ and $r_{a}\left(r_{s}<r_{a}\right)$. The other symbols represent observational constraints for the fast solar wind taken from McComas et al. (2000) for $U, n$ at $1 \mathrm{AU}$; Grall et al. (1996) for $U$ inside $1 \mathrm{AU}$; Breech et al. (2005) for $\sigma_{c}$ and proton temperature beyond $1 \mathrm{AU}$; Cranmer (2009) for proton temperature inside 1 AU; Banerjee et al. (1998) and Fisher \& Guhathakurta (1995) for $n$ inside 1 AU; and Bavassano et al. (2000) for the turbulence level.

(A color version of this figure is available in the online journal.)
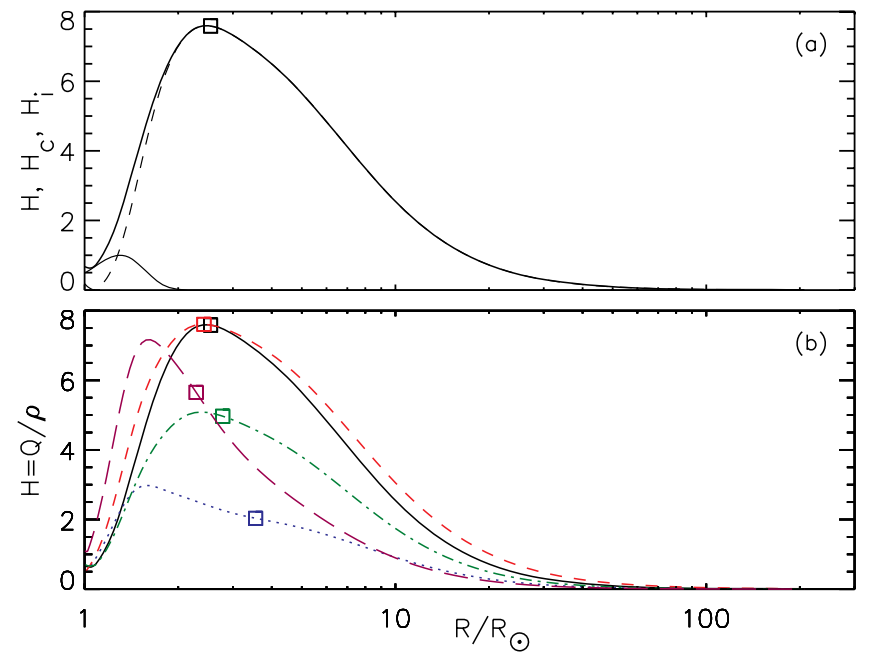

Figure 2. (a) Total (thick line), compressive (thin line) and incompressible (dashed line) heating function (dissipated energy per unit mass) as a function of heliocentric distance in units of $\approx 3 \times 10^{10} \mathrm{~cm}^{2} \mathrm{~s}^{-3}$ for the reference solution. (b) Total heating function for the solutions obtained varying the parameters as described in the caption of Figure 1 (same line and color coding). Empty squares mark the location of $r_{s}$ for each solution.

(A color version of this figure is available in the online journal.)

to the observed proton temperature. The latter is obtained by subtracting from the observed line width $w_{\perp}$ the contribution of the turbulent fluctuation $\delta u$, according to the relation ${ }^{7}$ $2 k_{B} T / m_{p}=w_{\perp}^{2}-(\delta u)^{2} / 2$ (e.g., Tu et al. 1998). The profiles of the fluctuation energy $E^{+}+E^{-}=Z^{2} / 2$ and normalized cross helicity $\sigma_{c}$ trend nicely toward the in situ data beyond $1 \mathrm{AU}$. The turbulent dissipation $H_{i}$ (Figure 2(a), dashed line) accounts for the spatially extended heating that accelerates the wind from below the sonic critical point. The compressive heating is small, about $1 \%$ of the total heating per unit mass. Its form is arbitrarily chosen to be a Gaussian (Figure 2(a), thin solid line), centered at $1.3 R_{\odot}$ with width $\approx 0.25 R_{\odot}$, in order to confine its contribution well below $r_{s}$.

\footnotetext{
7 Only the reference case is used to compute the corrected proton temperature in Figure 1. Other cases give similar values except the $\delta u_{\odot}=20 \mathrm{~km} \mathrm{~s}^{-1}$ and $H_{c}=5 \% H$ cases, yielding much higher corrected temperatures.
}

The solutions change as parameters are varied, but not all the parameters have the same impact. For example, for a steep spectrum of low-frequency waves $\left(\omega \lesssim 10^{-5} \mathrm{~Hz}\right.$, slope $\lesssim-1.1$ ), use of only zero-frequency fluctuations is a very good approximation. For flatter spectra, the high-frequency part of the spectrum remains principally outward propagating $\left[z^{+}(\omega)\right]$, thus limiting the total turbulent dissipation. This affects the turbulent energy $Z^{2}$, which becomes larger beyond $r_{a} \approx 13 R_{\odot}$, and also $\sigma_{c}$, which stays closer to unity. $U, T$, and $\rho$ are almost unchanged ( $U$ and $T$ are slightly reduced). Below, we consider only $\omega=0$ fluctuations.

The position of the sonic critical point depends upon momentum and heat addition (Leer et al. 1982). Given that we specify $A_{\odot}$ and $\rho_{\odot}$, the mass flux is determined by $U_{\odot}$, which is found by requiring that the solution becomes supersonic on passing through $r_{s}$. It follows that deposition of heat before $r_{s}$ increases $T\left(r_{s}\right)$ and typically $U_{\odot}$, and thus also the mass flux. Deposition of heat beyond $r_{s}$ does not alter $T\left(r_{s}\right)$, and so the mass flux is unchanged; however $U$ increases and $\rho$ decreases beyond $r_{s}$, with respect to a reference solution. Generally speaking, the maximum of $Z^{2}$ is below $r_{a}$ (it coincides with $r_{a}$ in the undamped case), the turbulent heating peaks near $r_{s}$, and the momentum added by the wave pressure has a maximum inside $r_{s}$.

Two ways of controlling the heat and momentum deposition (i.e., adjusting the importance of nonlinear terms relative to reflection/WKB (linear) terms in Equations (6)) are through variations of $\delta u_{\odot}$ and $\lambda(r)$. Decreasing the basal fluctuation amplitude $\left(\delta u_{\odot}=20 \mathrm{~km} \mathrm{~s}^{-1}\right.$, in Figure 1) decreases the turbulent heating and acceleration. In addition, heat deposition peaks well below $r_{s}$ (Figure 2(b)), yielding a slow, overdense, cool wind, with a deficit of turbulent energy. It is clear that $H_{c}$ shapes the solution only below $1.5 R_{\odot}$ while differences arise from a different shape of $H_{i}$. The asymptotic $Z^{2}$ and $\sigma_{c}$ do not vary much since beyond $r_{a}$ reflection is negligible and the ratio $Z_{-} / Z_{+}$is then controlled by turbulent dissipation and $\lambda(r)$.

Modification of $\lambda(r)$ leaves unchanged the position of the critical points; i.e., it only slightly alters the turbulent heating close to the coronal base, where reflection controls the ratio $Z_{-} / Z_{+}$. A larger basal correlation scale $\left(\lambda_{\odot}=0.05 R_{\odot}\right.$ in Figure 1) affects the solution mainly beyond $r_{s}$, yielding a hotter and faster wind, but with the density almost unchanged. The fluctuations have features similar to the undamped solutions, 
resulting in excess turbulent energy (that peaks closer to $r_{a}$ ) and more inward propagating waves (a smaller $\sigma_{c}$ ). Nonetheless, the increased turbulent energy - driven by reflection and the WKB term-broadens the turbulent heating while keeping fixed its maximum (Figure 2(b)).

Adjusting the expansion $A(r)$ also changes the correlation scale through $\lambda(r)=\lambda_{\odot} \sqrt{A(r)} . A(r)$ controls the reflection term directly and through the Alfvén speed gradients (Equations (7) and (8)). Decreasing the maximum super-radial expansion, to $f_{\max }=10$, while keeping fixed its location at $r_{f} \approx 1.3 R_{\odot}<r_{s}$ (Figure 1 ) has two consequences. First, it decreases the correlation scale for $r \gtrsim r_{f}$, causing a reduction of turbulent energy and heating. Second, it increases the density scale height around $r_{f}$, hence reducing reflection and the amount of turbulent dissipation there. The result is much smaller turbulent heating that peaks again at $r_{s}$ (Figure 2(b)) yielding a slower, cooler, and denser wind, even as close as $r_{s}$, which retains good asymptotic $Z^{2}$ and $\sigma_{c}$.

Finally, let us examine the role of compressive heating, recalling that $H(r)=H_{c}+H_{i}$. If the compressive heating is increased to $H_{c} \approx 5 \% H$ (Figure 1, violet dashed), the temperature maximum moves closer to the coronal base, but the resulting wind is slower, denser, and cooler. An increased $H_{c}$ also alters the incompressible heating - the height-integrated heating is almost unchanged, but heat deposition occurs at lower $r$ due to increased reflection at $r<r_{s}$ (Figure 2(b), compare the smaller $\sigma_{c}$ in Figure 1). Beyond the peak of $H$ the fluctuation energy remains small, reducing the extended turbulent heating.

On the other hand, decreasing $H_{c}$ enhances the incompressible heating; then $H$ has a minimum near the maximum of $V_{a}$, just inside $r_{s}$, and also peaks outside $r_{s}$. This causes convergence problems. In early iterations, heat deposition is mainly outside $r_{s}$, yielding a very fast, underdense, and hot wind, with strong density gradient (and hence reflection) at $r>r_{s}$. This leads to runaway iterations in which the sonic point moves to larger $r$ with a temperature minimum inside $r_{s}$. When the temperature minimum becomes very low the sonic critical point is reached with $\mathrm{d} T / \mathrm{d} r<0$. Then steep gradients occur close to the coronal base, heat deposition occurs close to $r_{s}$, and the solution again resembles the initial solution, producing the ensuing runaway. To obtain a solution, the key features of $H_{c}$ are that it is localized well below $r_{s}$, and is at least $\sim 0.7 \%$ of the total heating (for other parameters at reasonable levels). We found that the results do not depend strongly on the form of the compressive heat function provided that this heating is localized and not too large (exponential, Gaussian, etc. all work).

\section{DISCUSSION}

The above model shows that turbulence near the coronal base, originating through chromospheric transmission of fluctuations, can heat the plasma in an expanding coronal hole flux tube and produce a fast solar wind that matches a number of observational constraints. The turbulence is mainly of the low-frequency Alfvénic type. A small amount of compressive heating between the transition region and the sonic point appears to be needed to match the observations. This additional heating may be due to type II spicules that supply broadband low-frequency vertical fluctuations at transition region heights, thus launching compressive MHD modes near the coronal base (DePontieu et al. 2009).

Most of the fluctuation energy is in low-frequency turbulence, and this sustains a strong anisotropic MHD cascade through reflections from local density gradients. This type of anisotropic cascade is favored in MHD turbulence in the presence of a strong DC magnetic field (Robinson \& Rusbridge 1971; Shebalin et al. 1983; Oughton et al. 1994). Heat conduction does not enter the present model at all, since it mainly affects electron internal energy, which evolves independently in this approximation. Similar assumptions work well in understanding observations of solar wind turbulence (Breech et al. 2009; Cranmer et al. 2009). In these ways, the present model differs from other recent models that incorporate turbulent heating (Suzuki \& Inutsuka 2005; Cranmer et al. 2007). In particular, we believe that this model demonstrates, possibly for the first time, that a model (almost) free of ad hoc heat functions, artificial equations of state, and ad hoc assumptions about heat conduction can indeed heat the corona and accelerate the solar wind.

We plan further study of the required small amount of compressible heating, attributed here to spicule-driven magnetosonic activity. Another useful extension would be to include separate electron and proton internal energy budgets, which will enable additional observational constraints, and will permit study of the role of kinetic dissipation processes (Breech et al. 2009; Cranmer et al. 2009).

This research was supported in part by NASA (Heliophysics Theory, NNX08AI47G) and NSF (Solar-Terrestrial and SHINE programs ATM-0539995 and ATM-0752135). A.V. acknowledges support from the Belgian Federal Science Policy Office through the ESA-PRODEX program. We acknowledge the UVCS project (J. Kohl, S. Cranmer) for providing data used in Figure 1.

\section{REFERENCES}

Banaszkiewicz, M., Axford, W. I., \& McKenzie, J. F. 1998, A\&A, 337, 940 Banerjee, D., Teriaca, L., Doyle, J. G., \& Wilhelm, K. 1998, A\&A, 339, 208 Bavassano, B., Pietropaolo, E., \& Bruno, R. 2000, J. Geophys. Res., 105, 15959 Belcher, J. W., \& Davis, L., Jr. 1971, J. Geophys. Res., 76, 3534

Bieber, J. W., Wanner, W., \& Matthaeus, W. H. 1996, J. Geophys. Res., 101, 2511

Breech, B., Matthaeus, W. H., Cranmer, S. R., Kasper, J. C., \& Oughton, S. 2009, J. Geophys. Res., 114, A09103

Breech, B., et al. 2005, Geophys. Res. Lett., 32, 6103

Cho, J., Lazarian, A., \& Vishniac, E. T. 2002, ApJ, 564, 291

Coleman, P. J. 1968, ApJ, 153, 371

Cranmer, S. R. 2009, LRSP, 6, 3

Cranmer, S. R., Matthaeus, W. H., Breech, B. A., \& Kasper, J. C. 2009, ApJ, 702, 1604

Cranmer, S. R., \& van Ballegooijen, A. A. 2005, ApJS, 156, 265

Cranmer, S. R., van Ballegooijen, A. A., \& Edgar, R. J. 2007, ApJS, 171, 520 de Kármán, T., \& Howarth, L. 1938, Proc. R. Soc. London A, 164, 192

DePontieu, B., McIntosh, S. W., Hansteen, V. H., \& Schrijver, C. J. 2009, ApJ, 701, L1

Dmitruk, P., Milano, L. J., \& Matthaeus, W. H. 2001, ApJ, 548, 482

Dobrowolny, M., Mangeney, A., \& Veltri, P. 1980, Phys. Rev. Lett., 45, 144

Fisher, R., \& Guhathakurta, M. 1995, ApJ, 447, L139

Grall, R. R., et al. 1996, Nature, 379, 429

Grappin, R., Leorat, J., \& Pouquet, A. 1983, A\&A, 126, 51

Habbal, S. R., Esser, R., Guhathakurta, M., \& Fisher, R. R. 1995 , Geophys. Res. Lett., 22, 1465

Hansteen, V. H., Leer, E., \& Holzer, T. E. 1994, ApJ, 428, 843

Hansteen, V. H., Leer, E., \& Holzer, T. E. 1997, ApJ, 482, 498

Hollweg, J. V. 1986, J. Geophys. Res., 91, 4111

Hollweg, J. V., \& Johnson, W. 1988, J. Geophys. Res., 93, 9547

Holzer, T., \& Leer, E. 1980, J. Geophys. Res., 85, 4665

Hossain, M., Gray, P. C., Pontius, D. H., Matthaeus, W. H., \& Oughton, S. 1995, Phys. Fluids, 7, 2886

Kopp, R. A., \& Holzer, T. E. 1976, Solar Phys., 49, 43

Langangen, Ø., et al. 2008, ApJ, 679, L167

Leer, E., Holzer, T. E., \& Fla, T. 1982, Space Sci. Rev., 33, 161

Matthaeus, W. H., Zank, G. P., Oughton, S., Mullan, D. J., \& Dmitruk, P. 1999, ApJ, 523, L93

Matthaeus, W. H., et al. 2004, Geophys. Res. Lett., 31, 12803 
McComas, D. J., et al. 2000, J. Geophys. Res., 105, 10419

McKenzie, J. F., Banaszkiewicz, M., \& Axford, W. I. 1995, A\&A, 303, L45

Oughton, S., Priest, E. R., \& Matthaeus, W. H. 1994, J. Fluid Mech., 280, 95

Robinson, D. C., \& Rusbridge, M. G. 1971, Phys. Fluids, 14, 2499

Shebalin, J. V., Matthaeus, W. H., \& Montgomery, D. 1983, J. Plasma Phys., 29,525

Suzuki, T. K., \& Inutsuka, S. 2005, ApJ, 632, L49

Tu, C.-Y., Marsch, E., Wilhelm, K., \& Curdt, W. 1998, ApJ, 503, 475

Velli, M. 1993, Adv. Space Res., 14, 123
Verdini, A., Dmitruk, P., Matthaeus, W., Oughton, S., \& Velli, M. 2006, in SOHO-17, 10 Years of SOHO and Beyond, ed. H. Lacoste \& L. Ouwehand (ESA SP-617; Noordwijk: ESA), 150

Verdini, A., \& Velli, M. 2007, ApJ, 662, 669

Verdini, A., Velli, M., \& Buchlin, E. 2009a, Earth Moon Planets, 104, 121

Verdini, A., Velli, M., \& Buchlin, E. 2009b, ApJ, 700, L39

Withbroe, G. L. 1988, ApJ, 325, 442

Withbroe, G. L., \& Noyes, R. W. 1977, ARA\&A, 15, 363 